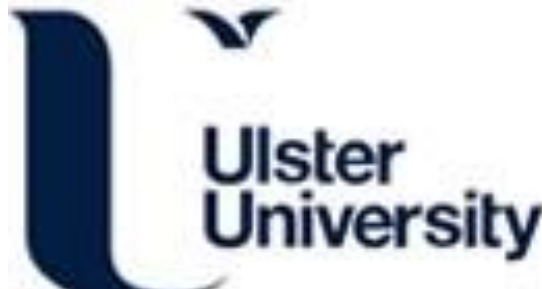

Home-Based Self-Management of Dementia: Closing the Loop

Patterson, T., Cleland, I., Hartin, P., Nugent, CD., Black, N., Donnelly, M., McCullagh, PJ., Zheng, H., \& McDonough, S. (2015). Home-Based Self-Management of Dementia: Closing the Loop. In Unknown Host Publication (Vol. 9102, pp. 232-243). Springer.

Link to publication record in Ulster University Research Portal

\section{Published in:}

Unknown Host Publication

Publication Status:

Published (in print/issue): 01/06/2015

\section{Document Version}

Publisher's PDF, also known as Version of record

\section{General rights}

Copyright for the publications made accessible via Ulster University's Research Portal is retained by the author(s) and / or other copyright owners and it is a condition of accessing these publications that users recognise and abide by the legal requirements associated with these rights.

\section{Take down policy}

The Research Portal is Ulster University's institutional repository that provides access to Ulster's research outputs. Every effort has been made to ensure that content in the Research Portal does not infringe any person's rights, or applicable UK laws. If you discover content in the Research Portal that you believe breaches copyright or violates any law, please contact pure-support@ulster.ac.uk. 


\title{
Home-Based Self-Management of Dementia: Closing the Loop
}

\author{
Timothy Patterson ${ }^{1}$, Ian Cleland ${ }^{1}$, Phillip J. Hartin ${ }^{1}$, Chris D. Nugent ${ }^{1}$, \\ Norman D. Black ${ }^{1}$, Mark P. Donnelly ${ }^{1}$, Paul J. McCullagh ${ }^{1}$, Huiru Zheng ${ }^{1}$, \\ Suzanne McDonough ${ }^{2}$ \\ ${ }^{1}$ School of Computing and Mathematics ${ }^{2}$ School of Health Sciences \\ University of Ulster, Newtownabbey, Co. Antrim, Northern Ireland, BT37 0QB \\ E-mail: \{t.patterson, i.cleland, cd.nugent, nd.black, mp.donnelly, \\ pj.mccullagh, h.zheng, s.mcdonough\}@ulster.ac.uk, \\ \{hartin-p1@email.ulster.ac.uk\}
}

\begin{abstract}
Within a technological home-based self-management of dementia paradigm there exists a nexus between a person with dementia, personalised reminding technology, detection of the adherence to reminders and an additional human-in-the-loop, for example a caregiver. To date, much work has focused on either the reminding technology per-se or monitoring adherence with less emphasis on integrating these aspects into a coherent self-management system for dementia. Within this paper, we present our current work on closing the remind-sensereason-act loop. The proposed framework is outlined and we describe an early-stage prototype that incorporates; (i) mobile-based reminding technology, (ii) detection of adherence by using environmental sensors and (iii) the potential to contact a carer in the event of non-compliance.
\end{abstract}

\section{Introduction}

It is estimated that between the years 2010 and 2050 the number of persons with dementia $(\mathrm{PwD})^{1}$ will increase over threefold from 35.56 million to 115.38 million [1]. This projected rise in worldwide prevalence is, in part attributed to increased life expectancy [2] coupled with the increasing levels of middleage obesity [3]. The promotion of self-management of chronic conditions is an emerging healthcare trend which aims to empower individuals to manage their illness in their own environment thereby, to some extent, alleviating the socioeconomic burdens imposed by such conditions. In the case of dementia this empowerment is achieved via approaches such as education, social engagement, collaborative goal setting between a PwD and health-care professional and the capture and display of health-related metrics, for example levels of activity.

In comparison to the other major chronic conditions such as Chronic Obstructive Pulmonary Disease, Coronary Heart Disease and Stroke, dementia primarily impacts upon a patient's mental ability by progressively reducing the

\footnotetext{
${ }^{1}$ Depending on context, PwD may also refer to a single person with dementia.
} 
capabilities of psychological functions such as the executive system and attention [4]. These symptoms may become increasingly severe eventually impacting upon the ability of a PwD to remember and perform Activities of Daily Living (ADLs). Consequently, one focus of our previous work [5] has been the development of mobile-based reminding technology to act as a cognitive prosthetic with the primary motivation of enabling a $\mathrm{PwD}$ to remain longer in their own homes.

There are two main reasons why a $\mathrm{PwD}$ should be afforded the opportunity to remain in their own home for as long as possible. Firstly, patients with mild dementia are likely to have a higher quality of life and level of social connectedness when receiving home-based as opposed to facility-based care [6]. Furthermore, where the caregiver is a family member the transition of a PwD between community and residential-based care may not decrease carer anxiety and in some cases actually leads to an increased risk of carer depression [7]. Secondly, from a financial perspective caring for a person with mild dementia in the community is considerably less expensive than the costs of care in a residential environment [8].

The system described in [5] reminds PwDs to perform ADLs such as taking medicine or eating regularly and requests that the user 'acknowledge' receipt of the reminder through simple interaction with the technology. Nevertheless, given the nature of dementia it is not sufficient to consistently assume that an acknowledged reminder is indicative of a $\mathrm{PwD}$ actually performing or completing the prompted task correctly. It is therefore necessary to consider alternative mechanisms for detecting reminder adherence for ADLs. Additionally, we consider the requirement of incorporating a human-in-the-loop, for example an informal caregiver, to provide intervention should episodes of non-compliance be detected.

The contribution of this paper lies in presenting a framework and an early stage prototype for facilitating home-based self-management of early-stage dementia. Specifically, we focus on closing the remind-sense-reason-act loop pertinent for our application resulting in a framework which contains mobile-based reminding technology, detecting adherence via environmental sensors and the potential to contact a human-in-the-loop, for example a caregiver. The remainder of this paper is structured as follows: in Section 2 we provide an overview of related work in the area of remind-sense-reason-act loop. The framework is presented in Section 3, with details of the early stage prototype discussed in Section 4. Finally, Conclusions and Future Work are outlined in Section 5.

\section{Related Work}

Whilst the problem of reminding a $\mathrm{PwD}$ to perform an activity is an active area of research, there has been less focus on integrating reminding technology, adherence detection and reasoning into a coherent self-management solution for dementia. Within this Section we provide an overview of related work pertinent to our target application specifically, reminding technology and detecting adherence. 


\subsection{Reminding technology}

Technological based reminding solutions have previously taken the form of pagers and voice reminders. NeuroPage [9] is an alphanumeric system, which sends short messages to a pager worn by a client. The pager is operated by a single button to acknowledge receipt of the reminder. Reminders are scheduled on a desktop PC which uploads the reminders to a central server where they are subsequently transmitted to the targeted pager device. Reminders are time and date based and usually relate to routine events such as taking medication, attending appointments, performing daily chores and meal preparation. Neuropage has been evaluated with over 200 patients with a range of cognitive impairments, including dementia and has been shown to be effective at assisting with perspective memory problems [10]. Due to technical limitations, however, such solutions have been criticised either for their lack of generalisability or the inability to modify the solution to suit the needs or preferences of the user [11].

More recently reminders have been delivered to a mobile platform in text format via email or Short Message Service (SMS). SMS reminders have been used within a number of health interventions with varying results. Previous studies have shown that SMS reminders can have a positive impact on medication adherence [12] and patient self-management [13]. A recent review by Kannisto et al. [14] found that although evidence for SMS application recommendations is still limited, the majority ( $77 \%$ ) of the studies thus far had shown improved health outcomes which may support its use in health care. The authors did note, however, that additional well-conducted SMS studies are required particularly in cohorts with varying age, gender, socio-economic backgrounds and conditions.

Although the use of SMS reminder systems within healthcare is still a relatively new concept [14], mobile technology continues to progress rapidly with UK smartphone ownership amongst memory impaired users reflecting that of the wider population [15]. Within the literature there are a number of smartphone applications reported which focus specifically on people with cognitive impairments, such as dementia. O'Neill et al. [16] developed a Mobile Phone Video Streaming (MPVS) system which provides video-based reminders for everyday activities. Videos are recorded by the carer or family member using a touch screen display and software application. The carer can schedule the reminders for the appropriate time and set the frequency with which they repeat. Upon receiving the reminder, users are required to acknowledge receipt by pressing a large button on a modified handset. This initialises the playback of the pre-recorded video. The application was developed through an iterative design process and has been evaluated with a cohort of $40 \mathrm{PwD}$ and their respective caregivers.

Reminder systems have attempted to improve reminder delivery by considering the context of the user (for example, user activity and location) in addition to time of delivery. Helal et al. [17] described a reminder system which uses data from sensors in the home to detect the location of the user and display reminder information, in the form of a video, on an appropriate display. Other reminder systems have attempted to utilise the user's location, detected from GPS or an 
indoor location system to deliver the reminder when the user is detected at a pre-defined location, for example escalate a reminder to buy milk when the user is at the local supermarket. The COGKNOW project [18] proposed a hybrid context-aware reminding framework for elders with mild Dementia (HYCARE). This architecture incorporated location and user activity as contexts, along with the time that the required activity was typically performed.

\subsection{Adherence Detection}

Few of the mobile reminder systems reported in the literature provide feedback on whether a task has been completed. A small number of the systems, for example Memojog [19] do provide functionality to alert a carer if the reminder is not acknowledged by the user. Nevertheless, user acknowledgement of a reminder does not necessarily mean that the user has interpreted the reminder and followed through and completed a task. Therefore methods to detect adherence to reminders has become an area of increased importance.

One solution to assess adherence of reminders is the use of activity recognition methods to accurately detect whether or not a scheduled activity has taken place. For example, computer vision algorithms make it possible to detect, where a person is and what they are doing within an environment. Radio-Frequency Identification (RFID) tags and accelerometer devices allow everyday objects such as cups and utensils to be uniquely labelled and then located. Data from environmental and body worn sensors can then be used to infer a user's activity. Systems have been developed which utilise environmental sensors to prompt the user during the completion of a task in order to aid task completion. The COACH system [20], for example, can monitor a user's progress washing their hands and prompt for completion only when necessary. These systems focus on complex step based activities, such as hand washing or preparing a meal and therefore differ in perspective from the reminder systems previously discussed.

Much of the work on adherence of reminders, assessing whether the individual has followed through and completed the task, has focused on medication reminders. In this research, the act of taking medication is typically inferred using either a simple binary result generated by a contact sensor or pressure sensor attached to a pill box, for example as described by Zhou et al. [21]. The indirect notion that a user takes their medication once it is removed from the container is largely naively accepted across these studies [22]. Asai et al. [23], designed a context aware medication reminder system. The system consisted of four modules: a sensing module, inferring module, ruling module and actuating module. The sensing module utilised a medication table consisting of a set of scales and an RFID receiver. When a medication bottle is removed from the scale, the system senses which bottle is moved using the RFID tags and can detect how many of the tablets are removed from the bottles by measuring the reduction in weight once the bottle is set back on the scales. The system uses a smartphone to deliver medication reminders and acts as a method for the user to self-report medication compliance whilst away from the home. 
O'Neill et al. [16] proposed a method of monitoring adherence to reminders through the use of low cost off-the-shelf sensors embedded within the environment. Sensors included contact sensors on doors and drawers, accelerometers on kettles and food containers and a buzzer for monitoring attendance at meetings. Sensor data was uploaded to a server where it was later mined using a bespoke analysis tool for evidence of task completion within a specific time of acknowledging a reminder. Results showed that low cost off-the-shelf sensors could be used to assess task completion following reminders, with $87 \%$ of tasks being sensed correctly. The authors suggested that such a system could provide peace of mind to PwDs and their respective carers by providing assurance that scheduled tasks have been completed. This provides a strong rationale to provide an end-to-end system which utilises low cost off-the-shelf sensors to provide feedback on task completion following reminders for PwDs.

\section{Framework}

Within this Section we present a framework for the self-management of dementia which incorporates a remind-sense-reason-act loop in the form of mobile-based reminding technology, detecting adherence and where appropriate contacting a human-in-the-loop.

In Figure 1, an overview of the four main components pertinent for homebased self-management of dementia are illustrated with the sequence of events shown in Figure 2. The 'Remind' component of our framework consists of a smartphone application (app) which enables a $\mathrm{PwD}$ or their caregiver to create, edit and delete reminders. When a reminder's date/time is reached a prompt is displayed on the smartphone with relevant details such as reminder type and description. There are two intuitive types of reminder response: acknowledged which indicates the $\mathrm{PwD}$ has read or conversely missed the reminder. The response is subsequently transmitted to cloud-based storage.

Giving consideration to the short-term memory impairment experienced by PwDs it is not sufficient to assume that the PwD has performed the displayed action. Therefore, taking into account the critical nature of specific types of reminders such as eating meals or taking medication upon a PwD's overall wellbeing it is necessary to detect adherence to the reminder. The 'Sensing' module of the framework consists of environmental sensors which would be statically placed around a PwD's home. Each sensor will have an associated location, for example 'medicine cupboard door', type, for example 'contact switch' and metric which denotes the types of reminder that a sensor is associated with, for example 'medicine'. Upon a change, a sensor's state is transmitted to a receiver where it is subsequently timestamped and uploaded to cloud-based storage.

A cloud-based system is used for storage of data and for executing the 'Reason' and 'Act' components. Whilst this results in the requirement of an available internet connection for uploading data it yields two main advantages from an extensibility perspective over performing the computation on hardware based in the PwD's home. Firstly, a cloud-based approach enables the 'Reason' and 'Act' 




Fig. 1. Self-Management of Dementia framework highlighting the main components of the remind-sense-reason-act loop.



Fig. 2. Sequence of data flow between system components. 
modules to be extended without updating software on devices within a PwD's home. Secondly, the approach readily facilitates the incorporation of internet connected sensors which do not require a dedicated home-based receiver.

Upon a reminder response being uploaded the 'Detect Adherence' module retrieves sensor observations for a given time frame and utilises the reminder type to determine reminder adherence. The 'Determine Action' module is subsequently called with reminder details such as the time and type, in addition to a boolean value indicating if the reminder was performed and an integer denoting the number of times the reminder was displayed. There are three possible outcomes of the 'Determine Action' module: flag reminder as performed, resend reminder and contact carer. Where the system has detected adherence, the reminder would be flagged as performed and the $\mathrm{PwD}$ would be oblivious to the cloud-based computation. Secondly, in the event of a missed reminder, or where it is detected that there has not been adherence, the reminder would be rescheduled for a user at a reminder-specific time in the future which reflects the estimated time required for the individual to perform the task.

Thirdly, upon detecting that there has been repeated (determined by an individual's condition) non-adherence of crucial reminders such as taking meals or medication a registered carer for the $\mathrm{PwD}$ would be contacted via a Google Cloud Message (GCM) delivered via a smartphone app. The carer would then interact with the $\mathrm{PwD}$ using, for example, a telephone call or personal visit. From a workflow perspective the primary advantage of incorporating a human-in-theloop in this manner is that a human such as a carer can quickly collate, analyse and act upon contextual information, for example the type of reminder and the PwD's recent history and subsequently act in an empathetic and intuitive fashion. Furthermore, from a carer's perspective the knowledge that a PwD is being unobtrusively monitored may decrease carer burden and anxiety [24].

\section{Prototype Implementation}

In this Section we present details of an early-stage prototype implementation of the discussed framework.

\subsection{Mobile-based reminding technology}

The smartphone reminder app has been developed to run on the Android platform, and acts as an assistive reminder tool for ADLs [5]. The developed app benefits from over 10 years of experience in the design, implementation and evaluation of assistive cognitive prosthetics, for example [16] [18]. An interdisciplinary team, consisting of computer scientists, psychologists, epidemiologists and statisticians designed the app through an iterative process and have evaluated its efficacy with a representative cohort [5]. The intended end users of the app are persons with the condition (in this case dementia) and their primary carers. The users can set temporal based reminders for any of the ADL types, for scheduled delivery at a specific time and date. The app follows a wizard 


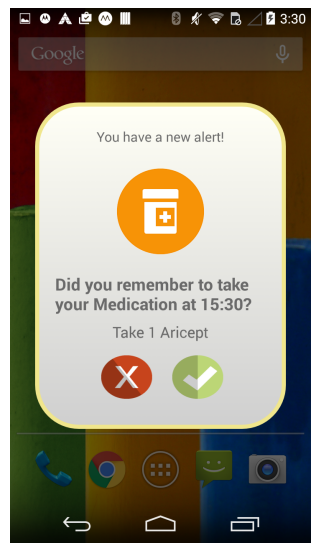

A Reminder prompt displayed on PwD's smartphone



B Repeat reminder prompt displayed on PwD's smartphone



C Alert displayed on a registered carer's smartphone

Fig. 3. Example screens from the PwD and carer smartphone applications.

design pattern, which guides the user through 6 sub-steps to create a reminder. Functionality to record voice messages has also been included.

To enable integration with the 'Act' component of the framework and to further promote the self-management of dementia the reminder app presented in [5] has been extended in two aspects. Firstly, we incorporate GCM enabling reminders to be automatically generated in response to non-adherence and pushed to the reminder app. Secondly, a characteristic of PwDs is that they may not be willing to acknowledge that they suffer from a memory impairment and therefore become agitated with a system that acts as a cognitive prosthetic [25]. With this in mind the reminder prompt is displayed as a question, for example instead of displaying "Remember to take your medication at 11:30" the reminder prompt would be rephrased to "Did you remember to take your medication at 11:30?" (Figure 3A).

The reminders can be configured to be repeated automatically on a daily or weekly basis. Repeat reminders are especially useful in scenarios in which a schedule is to be maintained, such as that imposed by a medication regimen [5]. A typical use case is a $\mathrm{PwD}$ living independently and having their carer or close family member set an array of reminders based on their understanding of the PwD's daily routine and needs. At the specified time, the reminder is delivered to the device as a popup accompanied by a melodic tone. The popup contains a textual description of the task to be performed, along with a graphic representing the ADL type (Figure 3A).

If the reminder is a voice message, the popup will indicate this to the user and a play button is presented. The message can be listened to as many times as is needed. In this application, the user has a default of 60 seconds in which to ac- 
knowledge the notification, before it is flagged as missed. This acknowledgement information is subsequently uploaded to cloud-based storage.

\subsection{Detecting Adherence}

The aim of the 'Detecting Adherence' module is to determine if the action associated with a specific reminder has been executed and incorporates the 'Sense' and 'Reason' components in Figure 1. From a sensing perspective there are three types of Tynetec sensors [26] utilised within the prototype implementation: a wall mounted passive infrared (PIR) sensor; magnetic contact switches placed on the room's two doors and a 'medicine' cupboard and, a pressure-activated sensor. Upon a change, each of the sensors transmit their state to a listener which subsequently timestamps the data and forwards it to cloud-based storage.

The 'Detect Adherence' module performs reasoning based on a specific reminder and relevant environmental sensor states. To accommodate the scenario where a $\mathrm{PwD}$ performs the task associated with a prompt before the reminder, an ADL and client-specific time duration is included when retrieving sensor states. Thus we consider sensor readings relevant if the state has changed in the time interval $\left[R_{T}\right.$-threshold, $\left.R_{T}\right]=\left\{X \in \mathbb{N} \mid R_{t}\right.$-threshold $\left.\leq X \leq R_{t}\right\}$ where $X$ is a non-negative integer containing a Unix timestamp, $R_{T}$ is the reminder time and threshold indicates an acceptable time for commencement of an activity before the reminder alert being displayed. At this stage in development the sensor states are combined in a rule-based manor resulting in a binary value indicating if the action associated with a reminder has been performed.

\subsection{Act}

The 'Act' component of the framework accepts a binary value indicating if the action associated with a reminder was performed and determines an appropriate action of either: resend reminder, contact carer or flag reminder as performed.

Should the activity associated with a reminder not be executed the system would in the first instance, resend the reminder to the client's device with the original reminder time incremented by an ADL specific value (Figure 3B). This value would be chosen at registration and reflects the time required for the $\mathrm{PwD}$ to perform the specific ADL. The repeat reminder is subsequently pushed to the reminder app using GCM. The number of times that a reminder may be resent is a user-defined parameter. Should the $\mathrm{PwD}$ repeatedly fail to perform the action associated with a reminder a carer such as a family member is contacted via an Android Carer App (Figure 3C). The Carer App receives a GCM containing the missed reminder details and upon the notification being clicked displays the details on screen. A 'call' button is incorporated which enables the carer to quickly telephone the $\mathrm{PwD}$. In the event of the $\mathrm{ADL}$ associated with a reminder being performed an 'adherence' log is updated and no further action is taken by the system. 


\subsection{Testing}

The prototype implementation was tested using three example ADLs of 'take medicine', 'appointment' and 'go to bed'. Nine reminders were set for each type for one healthy participant (27 reminders in total). The participant was instructed to perform each ADL as follows: 'take medicine' consisted of \{open door 1, enter room, open Medicine cupboard door\}, attend appointment consisted of $\{$ enter room, open door 2, exit room\} and 'go to bed' consisted of \{open door 1, enter room, activate pressure sensor\}.

The participant was instructed to perform the activity in advance of three reminders being displayed (i.e. Figure 2 condition 'Action Performed $==$ true'), perform the activity after three reminders had been displayed (i.e. Figure 2 condition 'No. Repeats $\leq$ Max. Repeats') and not perform the activity for three reminders (i.e. Figure 2 condition 'No. Repeats $>$ Max. Repeats'). The focus of testing was to verify that the appropriate system actions of 'flag reminder as performed', 'resend reminder' and 'contact carer' was executed as expected (Figure 2). This was a straight forward verification test of the three use cases. In these tests the system performed as expected with nine reminders flagged as acknowledged, nine reminders resent to the reminder app with the time incremented by a predetermined threshold and nine alerts sent to the carer app. Whilst these tests demonstrate the technical feasibility of the approach it is envisaged that future software development iterations will be validated using contemporary methodologies and tools orientated towards smart environments, for example the Spin based method presented by Augusto and Hornos [27].

\section{Conclusions and Future Work}

Within this paper we have presented a framework and early-stage prototype for the home-based self-management of dementia. In particular we have focused on closing the remind-sense-reason-act loop pertinent to our application resulting in an approach which incorporates reminding technology, environmental sensing, reasoning to detect adherence and an 'Act' component which determines an appropriate action to take. Early tests have been discussed which demonstrate the technical feasibility and practical potential of the approach.

There will be two main focuses of future work: firstly identification of ethical considerations, parameter choices such as the time taken to perform a task and visual design requirements will be solicited from $\mathrm{PwDs}$ and carers via a workshop. Secondly, from an implementation perspective the 'Sense' and 'Reason' components will be extended to detect adherence for further ADL types such as 'making a meal' or 'taking a drink' in multiple occupancy scenarios with the complete system validated using a pre-pilot cohort in a representative Smart Living Environment [28]. After further refinement the system will be trialled with a small cohort of PwDs and their associated caregivers thus enabling the identification of further real-world challenges and capture of performance and usage metrics. 


\section{Acknowledgements}

The authors gratefully acknowledge support from Invest Northern Ireland under Research and Development grant RD0513844. Additionally, we wish to thank Dr. Jonathan Synnott (Connected Health and Innovation Centre, University of Ulster) for assistance with the sensors used within this work.

\section{References}

1. M. Prince, R. Bryce, E. Albanese, A. Wimo, W. Ribeiro, and C. P. Ferri. The global prevalence of dementia: a systematic review and metaanalysis. Alzheimer's $\mathcal{E}$ dementia : the journal of the Alzheimer's Association, 9(1):63-75.e2, January 2013.

2. Aronson M. K., Ooi W. L., Geva D. L., Masur D., Blau A., and Frishman W. Dementia: Age-dependent incidence, prevalence, and mortality in the old old. Archives of Internal Medicine, 151(5):989-992, 1991.

3. R. A. Whitmer, E. P. Gunderson, E. Barrett-Connor, C. P. Quesenberry, and K. Yaffe. Obesity in middle age and future risk of dementia: a 27 year longitudinal population based study. BMJ (Clinical research ed.), 330(7504), June 2005.

4. C. L. Stopford, J. C. Thompson, D. Neary, A. M. T. Richardson, and J. S. Snowden. Working memory, attention, and executive function in Alzheimer's disease and frontotemporal dementia. Cortex, 48(4):429-446, April 2010.

5. P. J. Hartin, C. D. Nugent, S. I. Mcclean, I. Cleland, M. C Norton, C. Sanders, and J. T. Tschanz. A smartphone application to evaluate technology adoption and usage in persons with dementia. In 36th Annual International Conference of the IEEE Engineering in Medicine and Biology Society, pages 5389-5392, Chicago, USA, 2014. IEEE.

6. A. W. Nikmat, G. Hawthorne, and S. H. Al-Mashoor. The comparison of quality of life among people with mild dementia in nursing home and home care-a preliminary report. Dementia, pages 1-12, July 2013.

7. R. Schulz, S. H. Belle, S. J. Czaja, K. a McGinnis, A. Stevens, and S. Zhang. Long-term care placement of dementia patients and caregiver health and wellbeing. JAMA : the journal of the American Medical Association, 292(8):961-7, August 2004.

8. H. Crisp. Spotlight on Dementia Care. Number October. The Health Foundation, London, UK, 2011.

9. Oliver Zangwill Centre Neuropsychological Rehabilitation Accessed December 2014. NeuroPage. Online, http://www.neuropage.nhs.uk/, 2014.

10. B. A. Wilson, H. C. Emslie, K. Quirk, and J. J. Evans. Reducing everyday memory and planning problems by means of a paging system: a randomised control crossover study. Journal of Neurology, Neurosurgery \& Psychiatry, 70(4):477-482, 2001.

11. A. M. Seelye, M. Schmitter-Edgecombe, B. Das, and D. J. Cook. Application of cognitive rehabilitation theory to the development of smart prompting technologies. Biomedical Engineering, IEEE Reviews in, 5:29-44, 2012.

12. M. Vervloet, L. van Dijk, J. Santen-Reestman, B. van Vlijmen, M. L. Bouvy, and D. H. de Bakker. Improving medication adherence in diabetes type 2 patients through real time medication monitoring: a randomised controlled trial to evaluate the effect of monitoring patients' medication use combined with short message service (sms) reminders. BMC health services research, 11(1):5, 2011. 
13. T. de Jongh, I. Gurol-Urganci, V. Vodopivec-Jamsek, J. Car, and R. Atun. Mobile phone messaging for facilitating self-management of long-term illnesses. Cochrane Database Syst Rev, 12, 2012.

14. K. A. Kannisto, M. H. Koivunen, and M. A. Välimäki. Use of mobile phone text message reminders in health care services: A narrative literature review. Journal of medical Internet research, 16(10), 2014.

15. E. M. Migo, B. I. Haynes, L. Harris, K. Friedner, K. Humphreys, and M. D. Kopelman. mHealth and memory aids: levels of smartphone ownership in patients. Journal of Mental Health, 00(00):1-5, September 2014.

16. S. A ONeill, S. Mason, G. Parente, M. P. Donnelly, C. D. Nugent, S. McClean, B. Scotney, and D. Craig. Video reminders as cognitive prosthetics for people with dementia. Ageing International, 36(2):267-282, 2011.

17. S. Helal, C. Giraldo, Y. Kaddoura, C. Lee, H. El Zabadani, and W. Mann. Smart phone based cognitive assistant. In UbiHealth 2003: The 2nd International Workshop on Ubiquitous Computing for Pervasive Healthcare Applications, 2003.

18. R. J. Davies, C. D. Nugent, M. P. Donnelly, M. Hettinga, F. J. Meiland, F. Moelaert, M. D. Mulvenna, J. E. Bengtsson, D. Craig, and R. M. Dröes. A user driven approach to develop a cognitive prosthetic to address the unmet needs of people with mild dementia. Pervasive and Mobile Computing, 5(3):253-267, 2009.

19. K. Morrison, A. Szymkowiak, and P. Gregor. Memojog-an interactive memory aid incorporating mobile based technologies. In Mobile Human-Computer InteractionMobileHCI 2004, pages 481-485. Springer, 2004.

20. A. Mihailidis, J. N. Boger, T. Craig, and J. Hoey. The coach prompting system to assist older adults with dementia through handwashing: An efficacy study. BMC geriatrics, 8(1):28, 2008.

21. S. Zhou, C. H. Chu, Z. Yu, and J. Kim. A context-aware reminder system for elders based on fuzzy linguistic approach. Expert Systems with Applications, 39(10):94119419, 2012.

22. S. Vurgun, M. Philipose, and M. Pavel. A statistical reasoning system for medication prompting. Springer, 2007.

23. D. Asai, J. Orszulak, R. Myrick, C. Lee, J. F. Coughlin, and O. L. de Weck. Context-aware reminder system to support medication compliance. In Systems, Man, and Cybernetics (SMC), 2011 IEEE International Conference on, pages 3213-3218. IEEE, 2011.

24. M. Torkamani, L. McDonald, I. Saez Aguayo, C. Kanios, M. N. Katsanou, L. Madeley, P. D. Limousin, A. J. Lees, M. Haritou, and M. Jahanshahi. A randomized controlled pilot study to evaluate a technology platform for the assisted living of people with dementia and their carers. Journal of Alzheimer's disease : JAD, 41(2):515-23, January 2014.

25. D. C. Mograbi, C. P. Ferri, A. L. Sosa, R. Stewart, J. Laks, R. Brown, and R. G. Morris. Unawareness of memory impairment in dementia: a population-based study. International psychogeriatrics, 24(6):931-9, June 2012.

26. Tynetec. Tynetec - Telecare Devices, Accessed January 2015. Online, http:// http://www.tynetec.co.uk/telecare-devices, 2015.

27. J. C. Augusto and M. J. Hornos. Software simulation and verification to increase the reliability of Intelligent Environments. Advances in Engineering Software, 58:18-34, April 2013.

28. C. D. Nugent, M. D. Mulvenna, X. Hong, and S. Devlin. Experiences in the development of a smart lab. International Journal of Biomedical Engineering and Technology, 2(4):319-331, 012009. 